\title{
Self-Rehabilitation Guided by a Mobile Application After Anterior Cruciate Ligament Reconstruction Leads to Improved Early Motion and Less Pain
}

\author{
Constant Foissey, M.D., Mathieu Thaunat, M.D., Louka Bondoux, M.D., \\ Bertrand Sonnery-Cottet, M.D., and Jean-Marie Fayard, M.D.
}

\begin{abstract}
Purpose: To evaluate the adherence rate and the contribution of self-rehabilitation (SR) guided by a mobile application after anterior cruciate ligament reconstruction (ACLR) in combination with physical therapy sessions on early knee function. Methods: This study was a retrospective analysis of prospectively collected data from a single health care facility. All patients who underwent ACLR by a single surgeon from December 2019 to September 2020 were included. Two groups were formed and compared based on use of the mobile app: users ( $>10$ days of use) and nonusers $(\leq 10$ days of use). Outcomes included physical examination at 3 and 6 weeks postoperatively. Results: A total of 65 patients were analyzed: 19 in the nonuser group and 46 in the user group. Adherence rate was $91 \%$ at 10 days, $71 \%$ at 15 days, $62 \%$ at 21 days, and $44 \%$ at 45 days. At 3 weeks, the user group was 3.86 times [range 1.12 to 13.3] as likely to lock the quadriceps during gait with crutches and was 4.2 times [range 1.2 to 14.3] as likely to be pain free. There was a tendency to have less flexion contracture in the user group (17\% versus $32 \%, P=.32$ ). At 6 weeks, the differences leveled out, but the user group still had slightly better quadriceps locking during gait without crutches $(87 \%$ versus $79 \%, P=.46)$. Conclusions: SR guided by a mobile app combined with a standard rehabilitation protocol is correlated with better knee function at initial follow-up. Level of evidence: IV, therapeutic case series.
\end{abstract}

$\mathbf{E}^{1}$ ffective rehabilitation after anterior cruciate ligament reconstruction (ACLR) is one determinant of good clinical outcomes and faster return to sport. There is a higher risk of graft failure if rehabilitation is not carried out properly. ${ }^{1-4}$ Numerous rehabilitation protocols have been described, ${ }^{5}$ with the vast majority being under the supervision of a physical therapist. In 2020, Andrade et al. ${ }^{6}$ published a systematic review on the quality and applicability of existing clinical practice

From the Centre Orthopédique Santy, Lyon, France (C.F., M.T., L.B., B.SC., J-M.F.) and the Hôpital Privé Jean Mermoz, Ramsay-Générale de Santé, Lyon, France (C.F., M.T., L.B., B.S-C., J-M.F.).

The authors report the following potential conflicts of interest or sources of funding: M.T. reports personal fees and other, Doctup; other, Arthrex. B.S-C. reports personal fees, Arthrex. J-M.F. reports personal fees and other, Doctup; other, Arthrex, Newclip, Xnov. Full ICMJE author disclosure forms are available for this article online, as supplementary material.

Received January 20, 2021; accepted July 11, 2021.

Address correspondence to Constant Foissey, M.D., Centre Orthopédique Santy, 24 avenue Paul Santy, 69008 Lyon, France.E-mail:constant.foissey@chu-lyon.fr

(C) 2022 THE AUTHORS. Published by Elsevier Inc. on behalf of the Arthroscopy Association of North America. This is an open access article under the CC BY-NC-ND license (http://creativecommons.org/licenses/by-nc-nd/4.0/).

2666-061X/2185

https://doi.org/10.1016/j.asmr.2021.07.007 guidelines after ACLR. They concluded that the programs were generally good but difficult to implement in daily clinical practice. Among their 3 highly recommended protocols, $2\left(\mathrm{MOON}^{7}\right.$ and $\left.\mathrm{APTA}^{8}\right)$ included a clearly defined home-based program.

The term self-rehabilitation (SR) includes all rehabilitation exercises performed autonomously at home without the supervision of a physical therapist. It has many advantages: it contributes to patient education by placing patients in the foreground of their health care, it allows the continuation of care when access to a physical therapist is not possible, and it can lower health care costs by spacing out physical therapy sessions. Hohmann et al. ${ }^{9}$ compared physiotherapy-guided versus home-based unsupervised rehabilitation after ACLR and found no difference at 12 months between the 2 groups. This echoes the work of Grant et al., ${ }^{10}$ who found better results in range of motion (ROM) at 3 months after SR.

The main limit of SR is the patients' diligence. ${ }^{11}$ Many factors that reduce patient adherence to home-based training have been identified. The lack of timely feedback and real-time supervision by a health care professional in an at-home setting are often cited as the most important factors. Also, because of lack of 
motivation and supervision, patients could perform exercises incorrectly, which increases the risk of reinjury. To counter that, many mobile applications for patient education have emerged over the past 5 years in all specialties combined ${ }^{12-14}$; however, few studies have really evaluated the adherence rate for those programs. $^{15}$

The purpose of this study was to evaluate the adherence rate and contribution of SR guided by a mobile app after ACLR in combination with physical therapy sessions on early knee function. Our hypothesis was that increased app utilization would correlate with better early postoperative functional recovery.

\section{Methods}

The study was approved by our hospital's research and ethics committee and was performed in accordance with the Declaration of Helsinki's ethical standards. Informed consent was obtained from all participants before enrollment in the study.

A retrospective analysis of prospectively collected data from a single-center database was performed. All patients operated on from December 2019 to September 2020 by a single senior surgeon (J.M.F.) and reviewed in person by a single independent practitioner (L.B., nonsurgical sports medicine physician) at the 3- and 6week follow-up examinations were included. In our center, patients are randomly reviewed by 4 different sports medicine physicians. Inclusion criteria were all patients undergoing primary ACLR with or without meniscus repair, meniscectomy, or lateral extraarticular reconstruction. Exclusion criteria were inability to use a mobile app or additional surgical procedures (osteotomy); lateral extra-articular reconstruction and meniscus repair were not excluded.

\section{Standard Rehabilitation}

Patients were asked to mobilize their knee without a brace, bearing weight with crutches immediately after surgery; active and passive ROM were limited from $0^{\circ}$ to $90^{\circ}$ in the first 3 weeks. Physical therapy sessions were started 10 days after surgery to reduce the risk of hematoma and avoid excessive walking. Rehabilitation aims, content, and progression followed current clinical guidelines. The rehabilitation protocol was not different in case of meniscal repair or lateral extra-articular reconstruction. Our national health insurance reimburses 40 sessions of 30 minutes maximum for every patient who undergoes ACL reconstruction. The choice of specific exercises and therapeutic modalities was at the discretion of the physical therapist, guided by a cover letter written by our department, in which each exercise was explained based on the time after surgery. Physical therapists were free to give their own SR protocol.

\section{Mobile Application}

The mobile app DoctUp ${ }^{\circledR}$ (Healing SAS, Chassieu, France) (Fig. 1) was presented to patients during the preoperative consultation as a support to use in addition to the physical therapy sessions. This app was designed by the participating surgeons, with homebased exercises progressing over the course of the rehabilitation from day 1 to day 90 . It is available freely on the Google Play Store and Apple App Store. Each exercise is accompanied by a short video with instructions, aims, and potential errors.

\section{Clinical Evaluation}

Routine follow-up was performed by an independent, blinded, nonsurgical sports medicine physician (L.B.) 3 and 6 weeks after surgery. Pain (visual analog scale [VAS]), swelling, ROM, quadriceps isometric activation, and quadriceps locking during gait (with and without crutches) were evaluated first (Fig. 2). Significant flexion contracture was defined as a loss of extension $\geq 5^{\circ}$ measured by a goniometer (Fig. 2A). Then information about the visits with the physical therapist and the use of the mobile app was collected based on the patients' own reports. If the recovery was not as expected after 3 weeks, tips were given to the patient to improve it.

\section{Statistical Analysis}

Two groups were formed based on utilization of the app. Days of use are expressed by taking day 1 after surgery as the starting point; patients starting the app after day 10 were considered nonusers. App use was considered satisfactory after $>10$ days of consecutive use. This threshold was used because it corresponds to the usual start of physical therapy sessions in our practice. Thus, we compared those performing standard SR following their surgeon's recommendation to those performing SR guided by a mobile app.

The continuous variables were averaged and reported with standard deviation and range. Comparisons were made using independent $t$ tests for normally distributed variables and the Mann-Whitney $U$ test for non-normally distributed variables. Categorical variables were summarized by their percentages; comparisons were done using chi-square or Fisher's exact test. Significant results after univariate analysis were assessed by a binomial logistic regression model including all the confounding factors. Significance was set at $\alpha=0.05 ; P$ values $<0.05$ were considered statistically significant. The statistical analyses were performed with XLstat (version 2015.1, Addinsoft, Paris, France). VAS for pain was interpreted according to the patient acceptable symptom state (PASS) with a threshold of $2^{16}$; there was no PASS fixed for flexion contracture, as its mere presence is unacceptable. 
Fig 1. Mobile application Doctup ${ }^{\circledR}$. Each exercise is accompanied by a short video with instructions, aims, and potential errors.
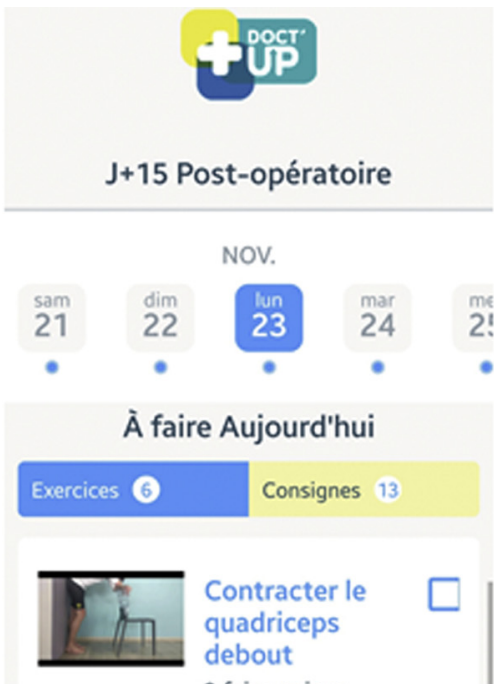

Contracter le quadriceps debout 3 fois par jour

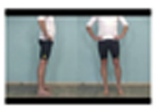

Verrouillage du genou en charge 3 fois par jour

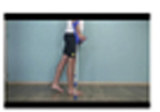

Le pas antérieur 3 fois par jour
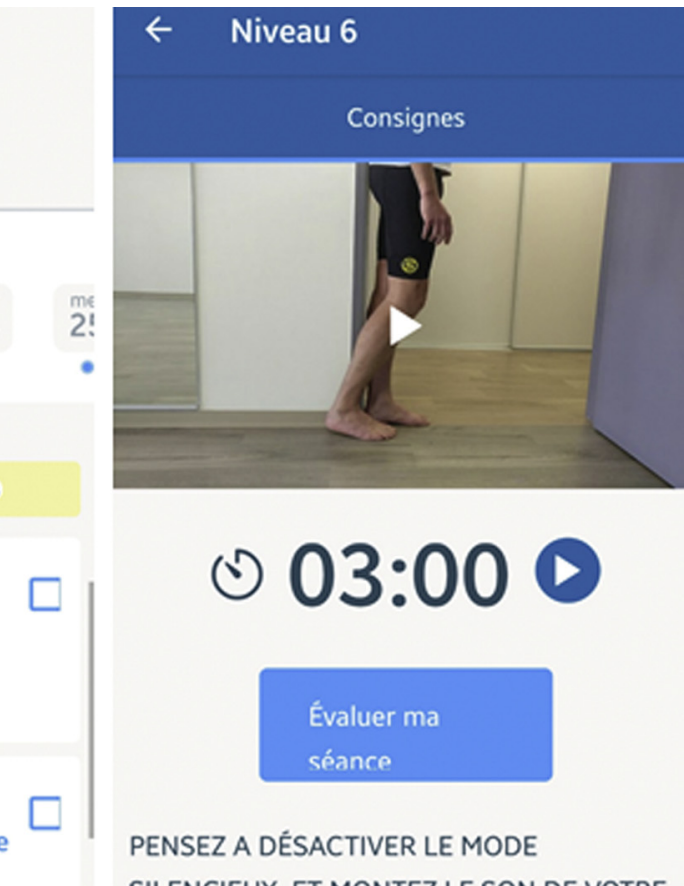

\section{3:00}

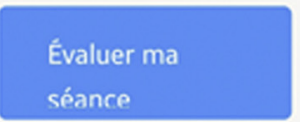

PENSEZ A DÉSACTIVER LE MODE

SILENCIEUX ET MONTEZ LE SON DE VOTRE TELEPHONE

() 3 minutes

3 fois par jour

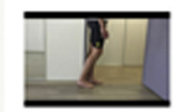

Le pas postérieur 3 fois par jour

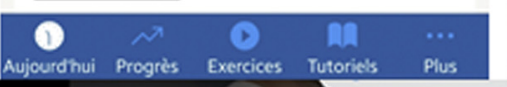

tous les jours à partir du 12ème jour
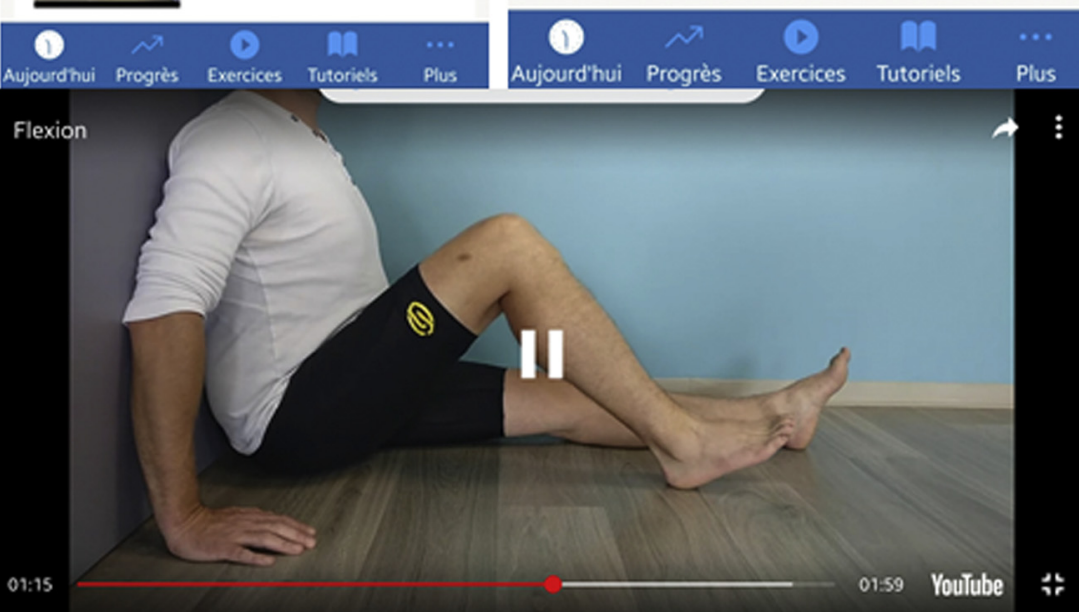

\section{Results}

Sixty-five patients met the inclusion criteria; no patients were excluded. No patients were lost to follow-up at 3 and 6 weeks. Nineteen patients used the mobile app for $\leq 10$ days, whereas 46 used it for $>10$ days. Patient demographics, details about the operation, and the physical therapist's care are summarized in Table 1. There were no significant differences between the 2 groups in these parameters.

\section{Use of the Mobile Application}

Six patients did not use the app at all $(91 \%$ use at 10 days), 13 stopped at day 10 (71\% use at 15 days), 6 stopped at day 15 (62\% use at 21 days), and 12 stopped at day 21 (44\% use at 45 days) (Fig. 3). Among the patients who did not use it at all, 5 forgot to use it from the very start and 1 had a connection problem. Among the patients who gave up before 10 days, 11 stopped at the beginning of the physical therapy sessions, 1 felt 

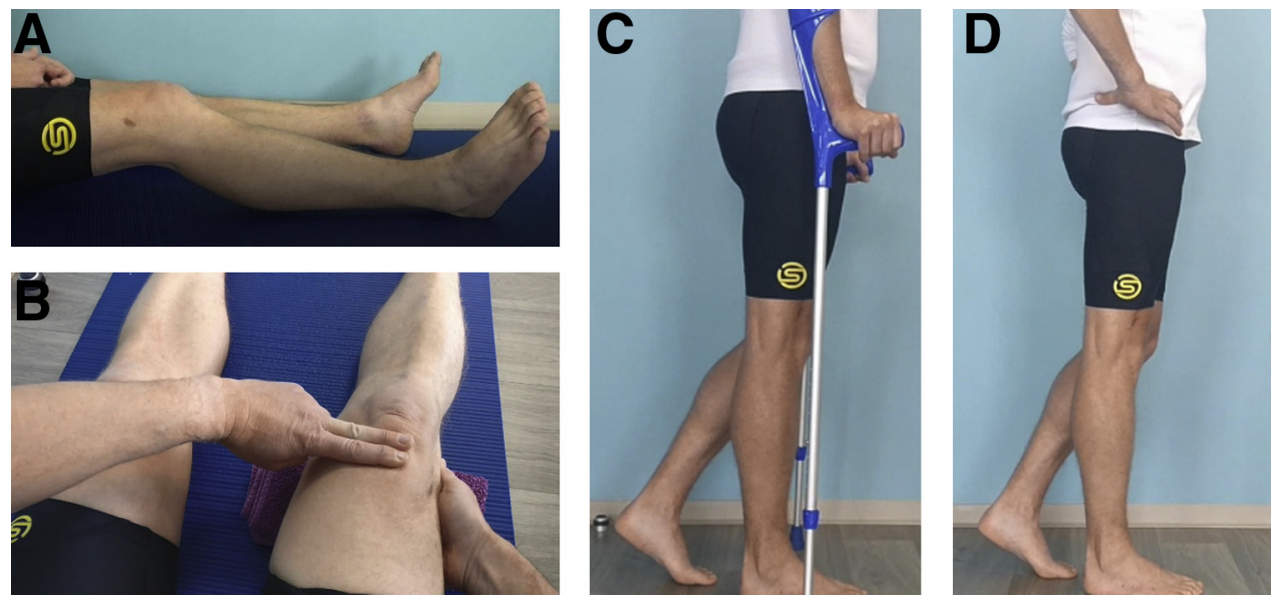

Fig 2. Clinical assessment. (A) Presence of a flexion contracture $\geq 5^{\circ}$. (B) Good quadriceps isometric activation assessed by ascension of the patella. (C) Good quadriceps locking with no flexion contracture at the maximum extension phase during gait with crutches. (D) Good quadriceps locking with no flexion contracture at the maximum extension phase during gait without crutches. that he had mastered the movements, and 1 felt the app was not diversified enough.

\section{Clinical Outcome}

Clinical outcomes are summarized in Table 2. No complications were reported during the entire followup period.

At 3 weeks, the share of patients recording some pain was significantly lower in the users than in the nonusers ( $17 \%$ versus $49 \%, P=.026)$. Mean VAS among the patients in pain was low: $1.2 \pm 0.4$ [range 1 to 2] for the nonusers and $1.4 \pm 0.7$ [range 1 to 3 ] for the users $(P=.535)$. The nonusers were twice as likely to have a flexion contracture $(32 \%$ versus $17 \%, P=.32)$. Mean flexion contracture was $6.3^{\circ} \pm 2.5^{\circ}$ [range 5 to 10] for the nonusers and $4.7^{\circ} \pm 0.7^{\circ}$ [range 3 to 5] for the users, $P=.51$ ). As for quadriceps activation, there was a significant difference in favor of the app users in ability to lock the quadriceps during gait with crutches. Those 2 differences continued to be significant after the multivariate analysis (Tables 3 and 4); use of the mobile app reduced by 4.2 the risk of having pain and multiplied by 3.9 the chance of being able to lock the quadriceps during gait with crutches.

At 6 weeks, all those differences leveled out. There was satisfactory flexion and elimination of flexion contracture in both groups. There was a tendency for better quadriceps locking during gait without crutches in the user group $(79 \%$ versus $87 \%$, $P=.46)$.

Table 1. Demographic, Intraoperative, and Postoperative Data Relative to Use of the Mobile Application

\begin{tabular}{|c|c|c|c|}
\hline Characteristic & $\leq 10$ days of use $(n=19)$ & $>10$ days of use $(n=46)$ & $P$ Value \\
\hline Age (y) & $29 \pm 10[14$ to 44$]$ & $29 \pm 12[15$ to 67$]$ & .91 \\
\hline Female & $7(37)$ & $21(46)$ & .59 \\
\hline BMI $\left(\mathrm{kg} / \mathrm{cm}^{2}\right)$ & $25 \pm 4[18$ to 31$]$ & $24 \pm 4[18$ to 35$]$ & .36 \\
\hline \multicolumn{4}{|l|}{ Type of graft } \\
\hline Hamstring & $18(95)$ & $42(91)$ & 1 \\
\hline Bone-patella tendon-bone & $1(5)$ & $4(9)$ & \\
\hline Suture & $5(26)$ & $15(32)$ & .77 \\
\hline Meniscectomy & $5(26)$ & $9(19)$ & .53 \\
\hline No treatment & $3(16)$ & $2(4)$ & .14 \\
\hline Osteochondral lesion & $6(32)$ & $12(26)$ & .76 \\
\hline ICRS grade of lesions & $2 \pm 0[2$ to 2$]$ & $2 \pm 0[2$ to 2$]$ & 1 \\
\hline Beginning of physical therapy sessions (d) & $10 \pm 1.6[7$ to 15$]$ & $9 \pm 2.3[7$ to 10$]$ & .07 \\
\hline 3 weeks & $6 \pm 4.6[0$ to 10$]$ & $20 \pm 2[14$ to 21$]$ & \\
\hline 6 weeks & $6 \pm 4.6[0$ to 10$]$ & $33 \pm 13[14$ to 45$]$ & \\
\hline
\end{tabular}

NOTE. Data are mean \pm SD [range] or $\mathrm{n}(\%)$.

BMI, body mass index; ICRS, International Cartilage Repair Society; SD, standard deviation. 
Fig 3. Adherence rate at days 10 , 15, 21 , and 45 .

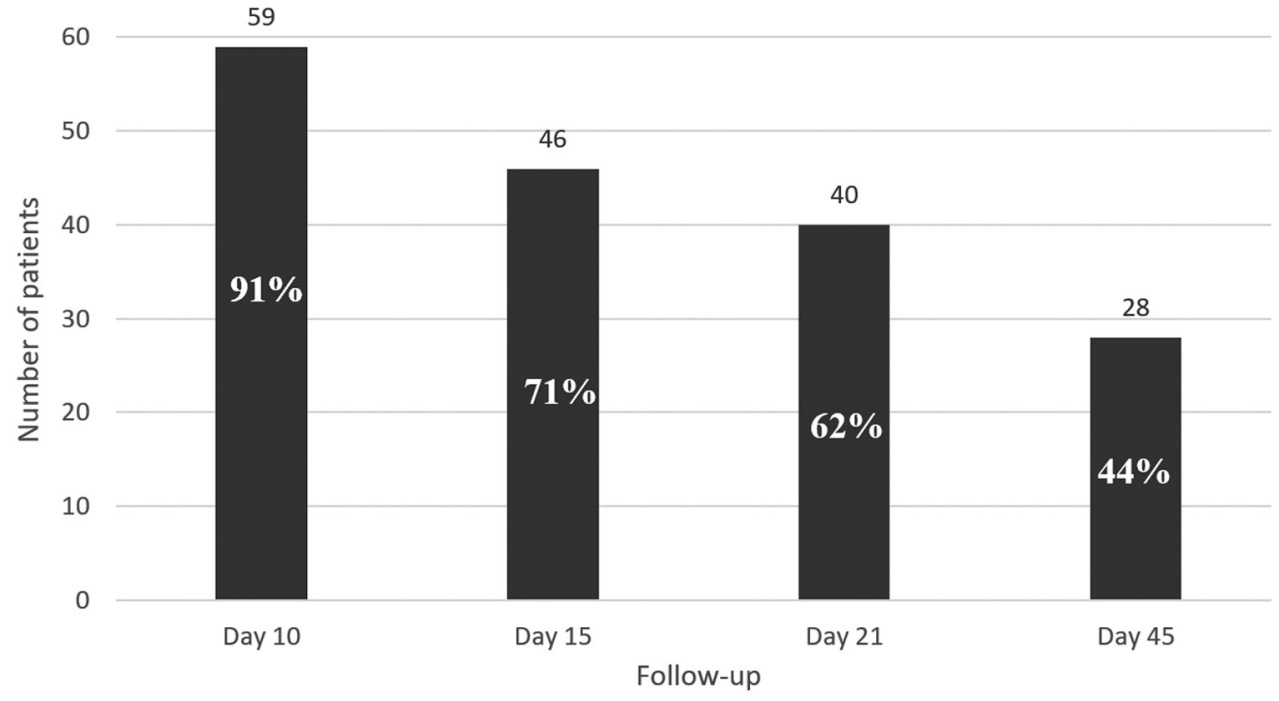

\section{Discussion}

The main finding of this study is the better early knee function after ACLR when the standard rehabilitation protocol is combined with SR guided by a mobile app. At 3 weeks, patients had a 4-fold lower risk of having pain and a 4-fold higher risk of having better quadriceps activation. The adherence rate was $91 \%$ at day 10 , $71 \%$ at day $15,62 \%$ at day 21 , and $44 \%$ at day 45 .

Quadriceps awakening is a major concern after knee surgery. Even the slightest improvement is valuable, since quadriceps deficiency may lead to extension deficit, cyclops syndrome, ${ }^{17}$ quadriceps atrophy, ${ }^{18}$ poor function, ${ }^{18}$ dynamic instability, ${ }^{19}$ gait abnormality, ${ }^{20}$ persistent knee pain, and early osteoarthritis. ${ }^{21}$ For these reasons, work on arthrogenic muscle inhibition
(AMI) after knee surgery or trauma is vital. SonneryCottet et al. ${ }^{22}$ undertook a review on the interventions used to improve AMI and found that there was low-quality evidence for neuromuscular electrical stimulation and transcutaneous electrical nerve stimulation, and very low-quality evidence for ultrasound and vibration. Good compliance with SR is a simple option to control AMI; its use produces better quadriceps awakening, associated with a decrease in flexion contracture and less pain among frequent users. This effect of SR on muscular function is probably linked to repeated central neurological stimulation, as shown in the neurological field after stroke. ${ }^{23-28}$

Alasfour and Almarwani ${ }^{15}$ studied the effect of a mobile app on adherence to a home-based exercise

Table 2. Clinical Outcomes Relative to Use of the Mobile Application

\begin{tabular}{|c|c|c|c|}
\hline Outcome & $\leq 10 \mathrm{~d}$ of use $(\mathrm{n}=19)$ & $>10 \mathrm{~d}$ of use $(\mathrm{n}=46)$ & $P$ Value \\
\hline \multicolumn{4}{|l|}{ 3-week follow-up } \\
\hline Painful & $9(47)$ & $8(17)$ & $.03 *$ \\
\hline$\geq$ PASS $($ VAS $=2)$ & $2(10.5)$ & $2(4.3)$ & .57 \\
\hline Flexion contracture & $6(32)$ & $8(17)$ & .32 \\
\hline Flexion $\left({ }^{\circ}\right)$ & $100 \pm 10[80$ to 120$]$ & $97 \pm 12[60$ to 120$]$ & .26 \\
\hline Quadricep isometric activation & $17(89)$ & $46(100)$ & .08 \\
\hline \multicolumn{4}{|l|}{ 6-week follow-up } \\
\hline Painful & 0 & 0 & 1 \\
\hline Swelling & $3(16)$ & $8(17)$ & .89 \\
\hline Flexion contracture & $1(5)$ & $1(2)$ & .50 \\
\hline Flexion $\left({ }^{\circ}\right)$ & $126 \pm 18[80$ to 140$]$ & $122 \pm 11[85$ to 140$]$ & .06 \\
\hline Quadricep isometric activation & $19(100)$ & $46(100)$ & 1 \\
\hline
\end{tabular}

NOTE. Data are $\mathrm{n}(\%)$ or mean \pm SD [range].

PASS, patient acceptable symptom state; SD, standard deviation; VAS, visual analog scale.

*Statistically significant. 
Table 3. Multivariate Analysis on Pain at 3 Weeks

\begin{tabular}{|c|c|c|c|}
\hline Characteristic & Painful $(\mathrm{n}=17)$ & Painless $(\mathrm{n}=48)$ & Odds Ratio \\
\hline Age $(\mathrm{y})$ & $30 \pm 12[17$ to 53$]$ & $29 \pm 12[14$ to 67$]$ & $1.01[0.95$ to 1.08$]$ \\
\hline Female & $8(47)$ & $20(42)$ & $1.23[0.30$ to 4.98$]$ \\
\hline BMI $\left(\mathrm{kg} / \mathrm{cm}^{2}\right)$ & $24 \pm 5[19$ to 31$]$ & $24 \pm 4[18$ to 35$]$ & $0.96[0.79$ to 1.18$]$ \\
\hline Meniscal lesions & $9(53)$ & $30(62.5)$ & $0.48[0.12$ to 1.84$]$ \\
\hline Osteochondral lesions & $5(29)$ & $13(27)$ & $1.57[0.33$ to 7.55$]$ \\
\hline Physical therapy sessions & $5 \pm 2[1$ to 10$]$ & $5 \pm 2[0$ to 10$]$ & $1.06[0.82$ to 1.39$]$ \\
\hline
\end{tabular}

NOTE. Data are mean \pm SD [range] or $\mathrm{n}(\%)$.

BMI, body mass index; SD, standard deviation.

*Statistically significant.

program for patients with knee osteoarthritis and found encouraging results, with a $27 \%$ additional adherence rate in the group using the app versus the nonusers. Many protocols recommend $\mathrm{SR}^{7,8}$ after ACLR. Some studies even found it to be similar alone over standard rehabilitation, ${ }^{9,29,30}$ but none could answer this question, as they were prospective randomized studies. We opted for an evaluation that fits more with reality to assess the efficacy and adherence rate in a more suitable protocol. The mobile app indeed does not aim to replace physical therapy but rather to supplement it. Even though $71 \%$ of patients reached our threshold for a significant level of use, there is still progress to be made to enhance adherence. We foresee 3 windows of opportunity to impact adherence: introducing the app first at the start so patients use it at the very beginning; second, at the beginning of the physical therapy sessions at day 10; third, after the first consultation with the sports physician at 3 weeks.

Accessibility, low cost, and safety are the main advantages of a mobile app, mostly in our young and connected sports medicine population. In 2015, around 165,000 mobile health apps were available for purchase; in 2019, apps included 76 for orthopaedic sports medicine. ${ }^{31,32}$ Although $65 \%$ of those apps are related to patient education or exercise programs, Wong et al. ${ }^{32}$ were concerned about their content and evidence base, as the majority had no named medical professional involvement and none were scientifically evaluated. Fortunately, since 2015, more and more authors have assessed those mobile app. ${ }^{15,33-35}$ Ardrern et al. ${ }^{36}$ are launching a study evaluating the efficacy of their app delivering cognitive behavioral therapy to help athletes return to sport. Higgins et al. ${ }^{37}$ recently studied a mobile app for postoperative home monitoring after ACLR that is projected to replace conventional follow-up visits with self-assessments directly sent to the surgeon. They found equal satisfaction and outcomes at 6 weeks, with noticeable cost savings for the patient and the health care system. ${ }^{37}$ Our 2 apps could be complementary, allowing medical, cognitive-behavioral, and rehabilitation home monitoring.

\section{Limitations}

This study is not without limitations. First, there was a short period of data collection (6 weeks). We wanted to focus initially on the early effectiveness of the app, as this is the key point of the rehabilitation: to get through AMI as fast as possible. ${ }^{6}$ Second, the small number of patients is a limitation. Despite this, we obtained significant results, but one might wonder whether differences such as the rate of flexion contracture at 3 weeks

Table 4. Multivariate Analysis on Quadriceps Locking with Crutches at 3 Weeks

\begin{tabular}{|c|c|c|c|}
\hline & $\begin{array}{l}\text { No Quadriceps Locking With } \\
\text { Crutches }(\mathrm{n}=14)\end{array}$ & $\begin{array}{l}\text { Quadriceps Locking With } \\
\text { Crutches }(\mathrm{n}=51)\end{array}$ & Odds Ratio \\
\hline Age $(y)$ & $28 \pm 14[17$ to 67$]$ & $30 \pm 11[14$ to 55$]$ & $1.03[0.97$ to 1.11$]$ \\
\hline Female & $7(50)$ & $21(41)$ & $0.53[0.14$ to 1.95$]$ \\
\hline Time to surgery (mo) & $8 \pm 9[1$ to 37$]$ & $8 \pm 15[0.5$ to 102$]$ & $1.07[0.94$ to 1.22$]$ \\
\hline Meniscal lesions & $9(64)$ & $30(59)$ & $0.64[0.18$ to 2.34$]$ \\
\hline Osteochondral lesions & $15(29)$ & $3(21)$ & $1.10[0.23$ to 5.02$]$ \\
\hline
\end{tabular}

NOTE. Data are mean \pm SD [range] or $\mathrm{n}(\%)$.

BMI, body mass index; SD, standard deviation.

*Statistically significant. 
may have been significant with more patients. Third, patients who diligently use the mobile app may be the most conscientious patients, which constitutes selection bias, as they may have a better natural result without the app. The last limitation could be the retrospective and nonrandomized design of this study; this bias is reduced here by the prospective collection of data.

\section{Conclusions}

SR guided by a mobile app combined with standard rehabilitation is correlated with better knee function at initial follow-up.

\section{References}

1. Acevedo RJ, Rivera-Vega A, Miranda G, Micheo W. Anterior cruciate ligament injury: Identification of risk factors and prevention strategies. Curr Sports Med Rep 2014;13:186-191.

2. Kyritsis P, Bahr R, Landreau P, Miladi R, Witvrouw E. Likelihood of ACL graft rupture: not meeting six clinical discharge criteria before return to sport is associated with a four times greater risk of rupture. $\mathrm{Br} J$ Sports Med 2016;50:946-951.

3. Ebert JR, Edwards P, Yi L, et al. Strength and functional symmetry is associated with post-operative rehabilitation in patients following anterior cruciate ligament reconstruction. Knee Surg Sports Traumatol Arthrosc 2018;26: 2353-2361.

4. Grindem H, Snyder-Mackler L, Moksnes H, Engebretsen L, Risberg MA. Simple decision rules can reduce reinjury risk by $84 \%$ after ACL reconstruction: The Delaware-Oslo ACL cohort study. Br J Sports Med 2016;50: 804-808.

5. Makhni EC, Crump EK, Steinhaus ME, et al. Quality and variability of online available physical therapy protocols from academic orthopaedic surgery programs for anterior cruciate ligament reconstruction. Arthroscopy 2016;32: 1612-1621.

6. Andrade R, Pereira R, van Cingel R, Staal JB, EspregueiraMendes J. How should clinicians rehabilitate patients after ACL reconstruction? A systematic review of clinical practice guidelines (CPGs) with a focus on quality appraisal (AGREE II). Br J Sports Med 2020;54:512-519.

7. Wright RW, Haas AK, Anderson J, et al. Anterior cruciate ligament reconstruction rehabilitation: MOON guidelines. Sports Health 2015;7:239-243.

8. Logerstedt DS, Scalzitti D, Risberg MA, et al. Knee stability and movement coordination impairments: Knee ligament sprain revision 2017. J Orthop Sports Phys Ther 2017;47: Al-A47.

9. Hohmann E, Tetsworth K, Bryant A. Physiotherapyguided versus home-based, unsupervised rehabilitation in isolated anterior cruciate injuries following surgical reconstruction. Knee Surg Sports Traumatol Arthrosc 2011;19:1158-1167.

10. Grant JA, Mohtadi NGH, Maitland ME, Zernicke RF Comparison of home versus physical therapy-supervised rehabilitation programs after anterior cruciate ligament reconstruction: A randomized clinical trial. Am J Sports Med 2005;33:1288-1297.

11. Miller KK, Porter RE, DeBaun-Sprague E, Puymbroeck MV, Schmid AA. Exercise after stroke: Patient adherence and beliefs after discharge from rehabilitation. Top Stroke Rehabil 2017;24:142-148.

12. Kloster M, Babic A. Mobile VR-application for neck exercises. Stud Health Technol Inform 2019;262:206-209.

13. Rauwerdink A, Jansen M, de Borgie CAJM, et al. Improving enhanced recovery after surgery (ERAS): ERAS APPtimize study protocol, a randomized controlled trial investigating the effect of a patient-centred mobile application on patient participation in colorectal surgery. BMC Surg 2019;19:125.

14. Bhatheja S, Fuster V, Chamaria S, et al. Developing a mobile application for global cardiovascular education. J Am Coll Cardiol 2018;72:2518-2527.

15. Alasfour M, Almarwani M. The effect of innovative smartphone application on adherence to a home-based exercise programs for female older adults with knee osteoarthritis in Saudi Arabia: A randomized controlled trial. Disabil Rehabil 2020:1-8.

16. Tubach F, Dougados M, Falissard B, Baron G, Logeart I, Ravaud P. Feeling good rather than feeling better matters more to patients. Arthritis Rheum 2006;55: 526-530.

17. Pinto FG, Thaunat $M$, Daggett $M$, et al. Hamstring contracture after ACL reconstruction is associated with an increased risk of cyclops syndrome. Orthop J Sports Med 2017;5:2325967116684121.

18. Lindström M, Strandberg S, Wredmark T, FelländerTsai L, Henriksson M. Functional and muscle morphometric effects of ACL reconstruction. A prospective CT study with 1 year follow-up. Scand J Med Sci Sports 2013:23:431-442.

19. Felson DT, Niu J, McClennan C, et al. Knee buckling: Prevalence, risk factors, and associated limitations in function. Ann Intern Med 2007;147:534-540.

20. Lewek M, Rudolph K, Axe M, Snyder-Mackler L. The effect of insufficient quadriceps strength on gait after anterior cruciate ligament reconstruction. Clin Biomech 2002; 17:56-63.

21. Segal NA, Glass NA, Torner J, et al. Quadriceps weakness predicts risk for knee joint space narrowing in women in the MOST cohort. Osteoarthritis Cartilage 2010;18:769-775.

22. Sonnery-Cottet B, Saithna A, Quelard B, et al. Arthrogenic muscle inhibition after ACL reconstruction: A scoping review of the efficacy of interventions. Br J Sports Med 2019;53:289-298.

23. Niama Natta DD, Lejeune T, Detrembleur C, et al. Effectiveness of a self-rehabilitation program to improve upper-extremity function after stroke in developing countries: A randomized controlled trial. Ann Phys Rehabil Med 2021:64:101413.

24. Bonnyaud C, Gallien P, Decavel P, et al. Effects of a 6month self-rehabilitation programme in addition to botulinum toxin injections and conventional physiotherapy on limitations of patients with spastic hemiparesis following stroke (ADJU-TOX): Protocol study for a randomised controlled, investigator blinded study. BMJ Open 2018;8:e020915. 
25. Gracies J-M, Pradines M, Ghédira M, et al. Guided selfrehabilitation contract vs conventional therapy in chronic stroke-induced hemiparesis: NEURORESTORE, a multicenter randomized controlled trial. BMC Neurol 2019;19:39.

26. Cai S, Chen Y, Huang S, et al. SVM-based classification of sEMG signals for upper-limb self-rehabilitation training. Front Neurorobot 2019;13:31.

27. Marsal C, Gracies J-M, Dean C, Mesure S, Bayle N. Beliefs of rehabilitation professionals towards guided selfrehabilitation contracts for post stroke hemiparesis. Top Stroke Rehabil 2017;24:608-613.

28. Rinne $\mathrm{P}$, Mace $\mathrm{M}$, Nakornchai $\mathrm{T}$, et al. Democratizing neurorehabilitation: How accessible are low-cost mobilegaming technologies for self-rehabilitation of arm disability in stroke? PLoS One 2016;11:e0163413.

29. Fischer DA, Tewes DP, Boyd JL, Smith JP, Quick DC. Home based rehabilitation for anterior cruciate ligament reconstruction. Clin Orthop Relat Res 1998;347:194-199.

30. Schenck RC, Blaschak MJ, Lance ED, Turturro TC, Holmes CF. A prospective outcome study of rehabilitation programs and anterior cruciate ligament reconstruction. Arthroscopy 1997;13:285-290.

31. Goz V, Spiker WR, Brodke D. Mobile messaging and smartphone apps for patient communication and engagement in spine surgery. Ann Transl Med 2019;7:S163 (suppl 5).

32. Wong SJ, Robertson GA, Connor KL, Brady RR, Wood AM. Smartphone apps for orthopaedic sports
medicine-A smart move? BMC Sports Sci Med Rehabil 2015;7:23.

33. Bahadori S, Wainwright TW, Ahmed OH. Smartphone apps for total hip replacement and total knee replacement surgery patients: A systematic review. Disabil Rehabil 2020;42:983-988.

34. Choi Y, Nam J, Yang D, Jung W, Lee H-R, Kim SH. Effect of smartphone application-supported self-rehabilitation for frozen shoulder: A prospective randomized control study. Clin Rehabil 2019;33:653-660.

35. Harmelink KEM, Zeegers AVCM, Tönis TM, Hullegie W, Nijhuis-van der Sanden MWG, Staal JB. The effectiveness of the use of a digital activity coaching system in addition to a two-week home-based exercise program in patients after total knee arthroplasty: Study protocol for a randomized controlled trial. BMC Musculoskelet Disord 2017; 18:290

36. Ardern CL, Kvist J, Ardern C, et al. BAck iN the Game (BANG) - A smartphone application to help athletes return to sport following anterior cruciate ligament reconstruction: Protocol for a multi-centre, randomised controlled trial. BMC Musculoskelet Disord 2020;21:523.

37. Higgins J, Chang J, Hoit G, Chahal J, Dwyer T, Theodoropoulos J. Conventional follow-up versus mobile application home monitoring for postoperative anterior cruciate ligament reconstruction patients: A randomized controlled trial. Arthroscopy 2020;36:1906-1916. 